\title{
Towards a generalised dual-mesh hybrid LES/RANS framework with improved consistency
}

DOI:

10.1016/j.compfluid.2017.08.002

\section{Document Version}

Accepted author manuscript

Link to publication record in Manchester Research Explorer

\section{Citation for published version (APA):}

Tunstall, R., Laurence, D., Prosser, R., \& Skillen, A. (2017). Towards a generalised dual-mesh hybrid LES/RANS framework with improved consistency. Computers and Fluids, 157, 73-83.

https://doi.org/10.1016/j.compfluid.2017.08.002

\section{Published in:}

Computers and Fluids

\section{Citing this paper}

Please note that where the full-text provided on Manchester Research Explorer is the Author Accepted Manuscript or Proof version this may differ from the final Published version. If citing, it is advised that you check and use the publisher's definitive version.

\section{General rights}

Copyright and moral rights for the publications made accessible in the Research Explorer are retained by the authors and/or other copyright owners and it is a condition of accessing publications that users recognise and abide by the legal requirements associated with these rights.

\section{Takedown policy}

If you believe that this document breaches copyright please refer to the University of Manchester's Takedown Procedures [http://man.ac.uk/04Y6Bo] or contact uml.scholarlycommunications@manchester.ac.uk providing relevant details, so we can investigate your claim.

\section{OPEN ACCESS}




\title{
Towards a Generalised Dual-Mesh Hybrid LES/RANS Framework with Improved Consistency
}

\author{
R. Tunstall ${ }^{\mathrm{a}, *}$, D. Laurence ${ }^{\mathrm{a}}$, R. Prosser ${ }^{\mathrm{a}}$, A. Skillen $^{\mathrm{b}}$ \\ ${ }^{a}$ School of MACE, The University of Manchester, Manchester, M13 9PL, UK \\ ${ }^{b}$ Scientific Computing Department, STFC Daresbury Laboratory, Warrington, WA4 4AD, UK
}

\begin{abstract}
This paper presents a combined RANS and LES methodology, which automates the dual-mesh hybrid LES/RANS approach and circumvents the need for wall-functions. Unlike RANS, wall-resolved LES is still unaffordable for studying high Reynolds number complex flows in industry and requires much user expertise when turbulence features are unknown a priori. The present approach avoids these issues by concurrently solving the LES and unsteady RANS equations on separate meshes, which are adapted to each model and are overlapping over the entire domain.

The RANS solution guides the LES where its mesh is too coarse, and vice-versa where the LES is well-resolved. The driver- and driven-simulation locally swap roles automatically, depending on which one is deemed more reliable by a blending function. Consistency between the RANS solution and a temporal average of the LES solution is enforced by drift terms, whose strength depend on relaxation times-scales that are provided by the RANS model.

Predictions for fully-developed channel flows and the flow through periodic hills are shown to be in excellent agreement with reference data. The LES grids are deliberately too coarse for wall-resolved LES, while the independent RANS mesh uses high aspectratio cells to economically resolve the near-wall layer.
\end{abstract}

Keywords: Hybrid LES/RANS, Turbulence modelling, Wall-bounded flows, OpenFOAM

\section{Introduction}

There are many engineering problems, such as thermal fatigue in nuclear plant thermal-hydraulics systems [1], where CFD techniques need to provide predictions for fluctuating quantities. Though wall-resolved LES and DNS can provide such predictions, the computational cost of these techniques is typically unaffordable for industrial

\footnotetext{
${ }^{*}$ Corresponding author.

Email address: ryan.tunstall@me.com (R. Tunstall)
} 
users at present. This is particularly the case for wall bounded flows, where grid refinement is necessary to resolve turbulent structures which become progressively smaller as a wall is approached.

In large eddy simulations wall-functions offer a solution to this problem by imposing empirical results in near-wall regions, allowing a coarser grid to be used. Wall-functions typically assume the boundary layer satisfies an equilibrium law, and in some cases this can degrade predictions. As an example, the limitations of wall-functions for flows in thermal-hydraulics systems is demonstrated in [2].

RANS techniques can resolve the mean flow field and, compared to wall-resolved LES, have much less stringent near-wall grid spacing requirements. Hybrid LES/RANS methods can exploit this to reduce the computational cost of wall-resolved LES while having a lower degree of empiricism than wall-functions. For a detailed review of hybrid LES/RANS methods the reader is referred to Fröhlich and von Terzi [3].

Xiao and Jenny $[4,5,6]$ have proposed a dual-mesh hybrid LES/RANS technique which solves the URANS and LES equations on separate grids with drift terms to enforce consistency between solutions. An exponentially weighted average (EWA) of the LES fields is calculated during the simulation, which is assumed to be equivalent to Reynolds averaging. Where the LES is classified as being well-resolved, drift terms relax the RANS fields to the EWA of the LES fields and where the LES is classified as under-resolved, drift terms relax the EWA of the LES fields towards the RANS fields.

Compared to existing unified hybrid LES/RANS methods, such as detached eddy simulations (DES) [7], the approach better respects the physical difference between Reynolds averaged and filtered qualities. The approach also results in clean conditions at internal LES/RANS interfaces where forcing of the filtered fields is not required, unlike zonal approaches such as that proposed by Davidson and Peng [8].

The dual-mesh hybrid LES/RANS framework is further developed in the present work, focussing on how it can be used to avoid the near-wall grid refinement associated with wall-resolved LES. We propose a number of changes which improve consistency of solutions from the two grids, increase automation and improve the agreement of predictions with reference data. The number of case-specific free-parameters is reduced by expressing the relaxation time-scales in terms of turbulent time-scales and using a blending function to automatically turn the various drift terms on or off. Several refinements to the drift terms which enforce consistency are also proposed.

\section{Consistency of Velocity Fields in a Dual-Mesh Hybrid LES/RANS Frame- work}

For an incompressible flow the momentum and pressure equations can be written for filtered or Reynolds-Averaged quantities as

$$
\frac{\partial U_{i}^{*}}{\partial t}+\frac{\partial U_{i}^{*} U_{j}^{*}}{\partial x_{j}}=-\frac{1}{\rho} \frac{\partial p^{*}}{\partial x_{i}}+\nu \frac{\partial^{2} U_{i}^{*}}{\partial x_{j} \partial x_{j}}-\frac{\partial \tau_{i j}^{*}}{\partial x_{j}}+Q_{i}^{*}
$$




$$
\frac{1}{\rho} \frac{\partial^{2} p^{*}}{\partial x_{i} \partial x_{i}}=-\frac{\partial^{2}}{\partial x_{i} x_{j}}\left(U_{i}^{*} U_{j}^{*}+\tau_{i j}^{*}\right)+\frac{\partial Q_{i}^{*}}{\partial x_{i}}
$$

where $Q_{i}^{*}$ is a momentum drift term, which will later be used to enforce consistency between the filtered and Reynolds-averaged fields. In the case of a large eddy simulation, the quantities are filtered such that: $U_{i}^{*}=\overline{U_{i}}, p_{i}^{*}=\overline{p_{i}}, \tau_{i j}^{*}=\tau_{i j}^{r}$ and $Q_{i}^{*}=Q_{i}^{L}$. Whereas for an unsteady Reynolds-averaged Navier-Stokes simulation the quantities are: $U_{i}^{*}=\left\langle U_{i}\right\rangle, p_{i}^{*}=\left\langle p_{i}\right\rangle, \tau_{i j}^{*}=\left\langle u_{i} u_{j}\right\rangle$ and $Q_{i}^{*}=Q_{i}^{R}$. From here on in, a superscript $L$ is used to denote quantities calculated on the LES mesh and $R$ for those calculated on the RANS mesh.

We perform a multilevel decomposition [9] of the LES, such that the velocity field is expressed as the sum of an exponentially weighted averaged, plus resolved and modelled fluctuations about this EWA:

$$
U_{i}=\left\langle\overline{U_{i}}\right\rangle^{\mathrm{EWA}}+u_{i}^{\prime \prime}+u_{i}^{\prime}
$$

where

$$
u_{i}^{\prime \prime}=\overline{U_{i}}-\left\langle\overline{U_{i}}\right\rangle^{\mathrm{EWA}}
$$

are the resolved fluctuations about the EWA and the modelled residual velocity field is

$$
u_{i}^{\prime}=U_{i}-\overline{U_{i}}
$$

The exponentially weighted averaging operator for a quantity $\phi$ is defined as

$$
\langle\phi\rangle^{\mathrm{EWA}}(t)=\int_{-\infty}^{t}\left(\frac{1}{\Upsilon} \phi\left(t^{\prime}\right) e^{-\left(t-t^{\prime}\right) / \Upsilon} d t^{\prime}\right)
$$

where $\Upsilon$ is the time-scale of the exponentially weighted average. Leibniz' rule for differentiation under an integral can be used to show that Equation (6) is a solution of the following differential equation:

$$
\frac{d\langle\phi\rangle^{\mathrm{EWA}}}{d t}=\frac{1}{\Upsilon}\left(\phi-\langle\phi\rangle^{\mathrm{EWA}}\right)
$$

which allows the exponentially weighted average to be approximated to first-order as $[10]$

$$
\left.\langle\phi\rangle^{\mathrm{EWA}}\right|_{t_{n}}=\left.(1-\alpha) \phi\right|_{t_{n}}+\left.\alpha\langle\phi\rangle^{\mathrm{EWA},}\right|_{t_{n-1}}
$$

with $\alpha=1 /(1+\Delta t / \Upsilon)$ and where $n$ relates to the time step. Compared to a traditional time-average, the use of an exponentially weighted average reduces the length of time required for the average to initialise and it prioritises recent events. Furthermore the first order approximation in Equation (8) is convenient, since it requires the storage of results from only the previous timestep.

It is assumed that the time-scale $\Gamma$ is long enough for the exponentially weighted 
average to be approximately the same as a Reynolds-average. Drift terms in the solved equations are then used to enforce consistency between the EWA of the filtered velocity field on the LES grid and the Reynolds-Averaged velocity field on the RANS grid, such that:

$$
\left\langle\overline{U_{i}}\right\rangle^{\mathrm{EWA}} \approx\left\langle U_{i}\right\rangle
$$

Xiao and Jenny [4] propose that when a Reynolds stress transport RANS model is used, drift terms should be employed to enforce consistency between individual stress components:

$$
\left\langle\tau_{i j}\right\rangle^{\mathrm{EWA}} \approx\left\langle u_{i} u_{j}\right\rangle
$$

here $u_{i}$ are the RANS fluctuating velocity components and $\tau_{i j}$ are the stresses arising from resolved and modelled fluctuations about the filtered EWA velocity

$$
\tau_{i j}=u_{i}^{\prime \prime} u_{j}^{\prime \prime}+\tau_{i j}^{r}
$$

It is assumed here that the EWA corresponds to a long term average, similar to a RANS operator, so that the resolved stresses can be written as product of resolved velocity fluctuations, unlike the LES residual stresses which need to be defined as

$$
\tau_{i j}^{r}=\overline{U_{i} U_{j}}-\overline{U_{i}} \overline{U_{j}} \neq \overline{u_{i}^{\prime} u_{j}^{\prime}}
$$

where $u_{i}^{\prime}$ is the modelled SGS velocity fluctuation [11].

However, when an eddy viscosity model is used to compute the RANS solution, a relationship between the Reynolds stresses and mean rate of strain is assumed. The EWA stresses $\left\langle\tau_{i j}\right\rangle^{\text {EWA }}$ computed on the LES grid are not subject to such an assumption. To minimise the impact of the RANS model's eddy viscosity hypothesis on the overall hybrid LES/RANS solution, the consistency requirement in Equation (10) is dropped and instead drift terms are employed to enforce consistency between the turbulent kinetic energies:

$$
\frac{1}{2}\left\langle\tau_{i i}\right\rangle^{\mathrm{EWA}} \approx k^{R}
$$

To ease notation we define $k^{\mathrm{EWA}}=\frac{1}{2}\left\langle\tau_{i i}\right\rangle^{\mathrm{EWA}}$. The drift terms in Equations (1) and (2), and additional drift terms in the transport equations solved by the turbulence models, are then used to enforce the above consistency requirements.

\subsection{LES Drift Terms}

Where the LES is classified as under-resolved, drift terms in the LES equations affect relaxation of the exponentially weighted average of the filtered fields towards the corresponding Reynolds-averaged fields, while the drift terms in the RANS equations are inactive. The drift term for the filtered momentum and pressure equations is

$$
Q_{i}^{L}=\left(1-\sigma^{L}\right)\left[\frac{\left\langle U_{i}\right\rangle-\left\langle\overline{U_{i}}\right\rangle^{\mathrm{EWA}}}{\gamma_{l 1}}+\frac{G_{i}}{\gamma_{l 2}}\right]
$$


where $\gamma_{l 1}$ and $\gamma_{l 2}$ are relaxation time-scales, which determine how quickly consistency is achieved. The blending parameter $\sigma^{L}$ varies between 0 and 1 to indicate whether the LES is considered well-resolved locally and is introduced in the present work to make the framework more flexible than the simple switch used by Xiao and Jenny. A value of $\sigma^{L}=1$ indicates the LES is well-resolved and $\sigma^{L}=0$ indicates the LES is under-resolved. The relaxation time-scales and blending parameter will be discussed in more detail in the sections that follow. Note that this and subsequent drift terms require the interpolation of quantities between the LES and RANS grids.

The first term in $Q_{i}^{L}$ acts to relax the EWA of the filtered velocity towards the Reynolds-averaged velocity, while the second term adjusts the resolved fluctuations about the EWA. In the present work we focus on RANS eddy viscosity models, and thus enforce consistency of the turbulent kinetic energies. The EWA turbulent kinetic energy needs to be relaxed to its RANS counterpart by a drift term of the form:

$$
\frac{\partial k^{\mathrm{EWA}}}{\partial t}=\frac{k^{R}-k^{\mathrm{EWA}}}{\gamma_{l 2}}
$$

In the LES there is no transport equation for $k^{\mathrm{EWA}}$ and so this requirement must be enforced by adjusting other quantities. Xiao and Jenny [4] elect to adjust only the resolved fluctuations using

$$
G_{i}=\frac{k^{R}-k^{\mathrm{EWA}}}{k^{R}+k^{\mathrm{EWA}}}\left(\overline{U_{i}}-\left\langle\overline{U_{i}}\right\rangle^{\mathrm{EWA}}\right)
$$

where a relationship between the time rate of change in a single component of the filtered velocity field needed to affect the consistency requirement in Equation (15) ( $=\frac{\partial k^{\mathrm{EWA}}}{\partial t} \frac{\partial u_{i}^{\prime \prime}}{\partial k^{\mathrm{EWA}}} \frac{\partial \overline{U_{i}}}{\partial u_{i}^{\prime \prime}}-$ no summation of indices) is derived using the chain rule, and it is assumed that each component of velocity should be adjusted by its relative contribution to the total turbulent kinetic energy.

\subsubsection{Adjusting Both the Resolved and Modelled Fluctuations}

The stresses in Equation (11), thus the EWA turbulent kinetic energy, have both resolved and modelled contributions. The $G_{i}$ term only acts on the resolved scales, meaning that good consistency cannot be attained when the modelled scales make a significant contribution to the EWA turbulent kinetic energy. To address this issue, we instead propose that consistency of the EWA turbulent kinetic energy should be enforced by adjusting both the resolved fluctuations and the modelled subgrid scale turbulent kinetic energy. This is the first novelty of the present work.

We compute an EWA of the subgrid turbulent kinetic energy $k_{\mathrm{sgs}}^{\mathrm{EWA}}=\left\langle\tau_{i i}^{r}\right\rangle^{\mathrm{EWA}} / 2$ and premultiply the $G_{i}$ term by the proportion of $k^{\mathrm{EWA}}$ which is resolved

$$
G_{i}=\left(1-\frac{k_{\mathrm{sgs}}^{\mathrm{EWA}}}{k^{\mathrm{EWA}}}\right) \frac{k^{R}-k^{\mathrm{EWA}}}{k^{R}+k^{\mathrm{EWA}}}\left(\overline{U_{i}}-\left\langle\overline{U_{i}}\right\rangle^{\mathrm{EWA}}\right)
$$


The forcing of the resolved fluctuations is thus adjusted to account for their contribution to the EWA turbulent kinetic energy. The subgrid $k$ is then also adjusted. In the present work the LES residual stress tensor is computed using a one equation eddy viscosity model $[12,13]$, which solves a transport equation for the subgrid turbulent kinetic energy, with Van Driest damping. In the hybrid solver the production of subgrid $k$ is modified to:

$$
P_{k_{\mathrm{sgs}}}=P_{k_{\mathrm{sgs}}}^{\mathrm{model}}+\left(1-\sigma^{L}\right) \frac{k_{\mathrm{sgs}}^{\mathrm{EWA}}}{k^{\mathrm{EWA}}} \frac{k^{R}-k^{\mathrm{EWA}}}{\gamma_{r 2}}
$$

where $P_{k_{\mathrm{sgs}}}^{\text {model }}$ is the original form of the subgrid $k$ production term and the second term represents the additional production of subgrid $k$ needed to achieve consistency between the RANS and EWA turbulent kinetic energies. The $k$ consistency requirement is thus achieved by adjusting both resolved and modelled fluctuations in the LES according to their relative contributions to the total EWA turbulent kinetic energy. If the subgrid contribution is zero, the Xiao and Jenny formulation is recovered.

\subsection{RANS drift Terms}

Where the LES is classified as well-resolved, the drift terms in the RANS momentum and pressure equations affect relaxation of the Reynolds-averaged fields towards the EWA of the filtered fields, while the drift terms in the LES equations are inactive. The drift term in the RANS momentum equation is

$$
Q_{i}^{R}=\left(1-\sigma^{R}\right) \frac{\left\langle\overline{U_{i}}\right\rangle^{\mathrm{EWA}}-\left\langle U_{i}\right\rangle}{\gamma_{r 1}}
$$

where $\gamma_{r 1}$ is a further relaxation timescale and $\sigma^{R}=1-\sigma^{L}$.

The original proposal of Xiao and Jenny [4] suggests adding drift terms to the transport equations for both the RANS turbulent kinetic energy and dissipation rate (or indeed any length scale surrogate):

$$
\begin{gathered}
Q^{k}=\left(1-\sigma^{R}\right) \frac{k^{\mathrm{EWA}}-k^{R}}{\gamma_{r 1}} \\
Q^{\epsilon}=\left(1-\sigma^{R}\right) \frac{\left\langle\epsilon^{L}\right\rangle^{\mathrm{EWA}}-\epsilon^{R}}{\gamma_{r 1}}
\end{gathered}
$$

where $\epsilon^{L}$ is the LES dissipation rate computed as

$$
\epsilon^{L}=2 \nu \overline{S_{i j}} \overline{S_{i j}}-\tau_{i j}^{r} \overline{S_{i j}}
$$

and $\bar{S}_{i j}$ is the filtered rate of strain tensor

$$
\bar{S}_{i j}=\frac{1}{2}\left(\frac{\partial \overline{U_{i}}}{\partial x_{j}}+\frac{\partial \overline{U_{j}}}{\partial x_{i}}\right)
$$


Consistency of the turbulent dissipation rate is only enforced one-way; there is no drift term in the LES equations to explicitly enforce its consistency where the LES is underresolved. Furthermore, the dual-mesh hybrid LES/RANS technique is designed to be used with relatively coarse LES grids, which may not result in accurate predictions for the dissipation rate due to its strong dependence on the strain rate.

\subsubsection{Modifications to the RANS Drift Terms}

Experience suggests that the turbulent dissipation rate drift term can degrade predictions from the hybrid solver when using time-scales that are effective at enforcing consistency throughout the entire domain. If, in an area where the LES is considered well-resolved, the $k$ drift term is acting to reduce RANS $k$ (to make it consistent with the LES EWA $k$ ), simultaneously forcing the dissipation rate to a consistent value will prevent the excess RANS $k$ from being dissipated. Instead the excess RANS $k$ spreads through the wider domain. If spreading occurs into a zone where the LES drift terms are active, the increased levels of RANS $k$ are fed back in to the LES, leading to degraded overall predictions.

The second novelty of the present work is to remove the drift term in Equation (21) from the turbulent dissipation rate equation. The drift term in Equation (20) is then treated as an additional turbulent kinetic energy production term:

$$
P_{k}=P_{k}^{\text {model }}+\left(1-\sigma^{R}\right) \frac{k^{\text {EWA }}-k^{R}}{\gamma_{r 2}}
$$

where $P_{k}$ is the production of turbulent kinetic energy to be used in the model's transport equations and $P_{k}^{\text {model }}$ is the production term specified in the original formulation of the RANS model. In many popular RANS eddy viscosity models (e.g. $k-\varepsilon, v^{2}-f$, elliptic blending $k-\varepsilon-v^{2} / k$ ) the production term appears in the equations for all transported turbulent quantities, and so adjusting it will result in these other quantities also responding to the $k$ consistency requirements. This approach prevents the issues associated with having explicit forcing of the turbulent dissipation rate and the framework now has the same consistency requirements where the LES is well and underresolved. Note that unlike in the original formulation, the time-scale associated with the forcing of RANS $k$ can take a different value to that used to force the RANS velocity $\left(\gamma_{r 2} \neq \gamma_{r 1}\right)$.

\subsection{Automatic Activation of Drift Terms}

In the original framework, LES mesh resolution is manually assessed a priori. Drift term activation is then controlled by a step function, which assumes that beyond a specified wall distance the LES mesh is sufficiently fine for the solution to be considered well-resolved, that is analagous to:

$$
\sigma^{L}= \begin{cases}0 & \text { when } y<D \\ 1 & \text { when } y \geq D\end{cases}
$$


where $y$ is the cell's wall distance and $D$ is the wall distance beyond which the LES is assumed to be well-resolved.

It is desirable however for the drift terms to be switched automatically, without recourse to case specific parameters, and to vary in space \& time to reflect the prevailing local flow physics. In a paper subsequent to their original proposal, Xiao and Jenny [6] automatically activated the drift terms according to whether pure LES could be considered well-resolved at a given location. Predictions were less satisfactory than those obtained using the original wall-distance based step function. This is likely because activation of the LES drift terms should not be governed by whether the mesh is fine enough for pure LES to be considered locally well-resolved, but rather by whether pure LES is able to outperform unforced RANS. In particular, predictions from the dual-mesh approach are likely to be degraded if the LES solution is guided by RANS in areas where the flow exhibits mean flow unsteadiness that unforced RANS is unable to sustain; here marginally under-resolved LES (that can at least sustain unsteadiness) may be preferable.

An optimal distribution of $\sigma^{L}$ is therefore likely to be a function of both the LES grid resolution \& the RANS grid resolution, the specific turbulence models employed and the choice of discretisation schemes. In the present work we seek to develop a robust blending function that maintains the generality of the dual-mesh hybrid LES/RANS framework and allow it to provide accurate predictions using meshes that are much too coarse for wall-resolved LES.

The high computational cost of wall-resolved LES is associated with its need for near-wall grid refinement, which is ultimately a consequence of viscosity. The need for this mesh-refinement can therefore be avoided in the dual-mesh approach if the LES solution is guided by RANS in viscous affected regions. The overall coupling we employ can be summarised as follows. In fully-turbulent regions the RANS solution is forced to the EWA of the LES solution. The RANS solution in such areas will therefore contain information about any unsteadiness in the mean flow. The unforced RANS solution near-walls then takes account of this unsteadiness and is used to guide the near-wall LES solution.

In the context of RANS modelling, a local wall-distance based turbulent Reynolds number can be used to identify fully-turbulent and viscous affected regions [14]

$$
R e_{y}=\frac{\sqrt{k} y}{\nu}
$$

where $R e_{y}>200$ indicates a fully-turbulent region and $R e_{y}<200$ indicates a viscous affected region. A blending function can then be formulated as:

$$
\sigma^{L}=\frac{1}{2}\left(1+\tanh \left(\frac{R e_{y}-R e_{y}^{*}}{A}\right)\right)
$$

where $R e_{y}^{*}=200$ determines the turbulent Reynolds number at which the switchover occurs. The constant $A=10$ determines the sharpness of the blending function; overall 
predictions do not exhibit a significant sensitivity to this parameter and its present value has been found adequate for the test cases reported herein.

\subsection{Timescale of the Exponentially Weighted Average}

The time-scale, $\Upsilon$, in Equation (6) governs how much of the instantaneous filtered velocity field is represented in the EWA and how much is represented in resolved fluctuations about the EWA. In the limit of a a very short averaging time, the EWA will be very similar to the instantaneous filtered field; small turbulent structures will be present within the EWA itself. Where the LES is well-resolved, the RANS fields are relaxed to the EWA of LES fields. However, the RANS grid may locally be coarser than the LES grid, particularly in the streamwise and spanwise directions, and so small structures in the EWA of filtered fields could be lost during interpolation to the RANS grid.

In the limit of a very long averaging time, the EWA fields will be smooth and similar in appearance to a classic steady-state RANS solution. In the case of a statistically stationary flow this is acceptable. However, a very long averaging time will also damp out the unsteadiness of the coherent structures of many transient flows, which may prove problematic. Furthermore, the longer the averaging time, the longer it will take for the EWA of the filtered fields to initialise and to then respond to the action of the drift terms once they are activated. With larger values of $\Upsilon$ it will therefore take longer for consistency between the RANS and LES grids to be established.

To minimise both interpolation errors and the amount of simulation time needed for the consistency requirements to be realised, it is advisable to choose the smallest value of $\Upsilon$ that ensures that each of the LES EWA fields being made consistent does not contain features which are too small to be interpolated to the RANS grid. Experience of the method thus far suggests that setting $\Upsilon$ to 10 or more mean flow time-scales is likely to be sufficient. Note that there can be transient behaviour in both the LES EWA fields and the RANS fields; separate running averages should therefore be computed during runtime for the purposes of collecting flow statistics.

\subsection{Automatic Relaxation Timescales}

The drift terms are of the form 'change required in quantity to ensure consistency between solutions from the LES and RANS grids' divided by a relaxation time-scale, which determines how quickly consistency of the solutions will be achieved. Xiao and Jenny [4] specified that these time-scales should have values within fractional ranges of the time-scale of the EWA; there is uncertainty in what value these time-scales should take. Furthermore, for more complex geometries it is conceivable that different values will be needed in different parts of the domain to reflect the locally prevailing flow physics.

To overcome these issues, we compute the relaxation time-scales during the simulation by relating them to turbulent time-scales. Since the time-scales of turbulence can vary significantly throughout the domain and in time, we allow the relaxation timescales to vary in space and update them once per time-step. There are a wide range 
of turbulent time-scales that the relaxation time-scales could be expressed in terms of. The suggestions of de Laage de Meux et al. [15] and further experimentation has led to the following choices:

$$
\begin{gathered}
\gamma_{l 1}=\gamma_{r 1}=\max \left(C_{\gamma 1} \frac{k^{R}}{\epsilon^{R}}, d t\right) \\
\gamma_{l 2}=\gamma_{r 2}=\max \left(C_{\gamma 2} \frac{k^{R}}{\epsilon^{R}}, d t\right)
\end{gathered}
$$

where $C_{\gamma 1}=0.1, C_{\gamma 2}=0.01$ and LES time-scales are computed using RANS quantities interpolated onto the LES grid. The time-scales are clipped to the time-step to avoid convergence difficulties.

\subsection{Implementation in OpenFOAM}

The hybrid solver described above has been implemented in the open-source CFD toolbox OpenFOAM 2.3.x [16]. The RANS and LES momentum and pressure equations with drift terms are solved using the pressure implicit with splitting of operations (PISO) algorithm [17]. OpenFOAM includes an efficient mesh-to-mesh interpolation library which is used to interpolate fields between the RANS and LES grids, using a volume conservative technique. The toolbox and derived solvers incorporate parallel running through domain decomposition with communication using the message passing interface (MPI) and the interpolation library does not require a given spatial location to have cells on the same MPI process in the decomposed LES and RANS meshes.

The Launder-Sharma viscous sublayer resolving $k-\epsilon$ model [18] is initially used for the RANS mesh. Later we demonstrate how predictions from the revised dual-mesh hybrid LES/RANS framework can be further improved by using the more advanced elliptic blending $k-\varepsilon-v^{2} / k$ model [19], which provides better predictions for near-wall turbulence by accounting for wall-normal stress anisotropy. LES solutions are obtained using a one equation SGS eddy viscosity model $[12,13]$, which solves a transport equation for the subgrid turbulent kinetic energy. Van Driest damping is applied to the LES length scale near-walls.

All equations are discretised using second-order schemes for convective and diffusive terms with a second-order backwards time scheme. In each of the reported simulations the time-stepping ensures that the maximum Courant number remains below 1 . At the start of a hybrid LES/RANS simulation there will inevitably be significant differences between the RANS fields and the EWA of the LES fields, which causes the drift terms to have a large magnitude. To prevent this from causing convergence problems, a linear unit ramp function is applied to the drift terms over the first $2 \Upsilon$ of runtime, which has been found sufficient to allow a stable coupling between solutions from the hybrid solver's RANS and LES meshes to be established. As the simulation progresses,

consistency between the solutions improves which in turn results in the magnitude of the drift terms decreasing. 


\section{Results for a $R e_{\tau}=395$ Channel Flow}

Here we conduct studies of a fully-developed $R e_{\tau}=395$ flow through a channel of half height $\delta$, and provide a comparison to DNS data from Iwamoto et aal. [20, 21]. The domain size is $2 \pi \delta \times 2 \delta \times \pi \delta$ in size, with periodic conditions on the inflow and sides. The LES grid has $50 \times 60 \times 30$ points with constant spacing in the streamwise, wall-normal and spanwise directions respectively. It is deliberately designed to be under-resolved in near-wall regions; the first cell centre is a distance of $y^{+} \approx 6$ from the wall, with $\Delta x^{+} \approx 50$ and $\Delta z^{+} \approx 41$ in the streamwise and spanwise directions respectively. The low-Reynolds number RANS grid has $10 \times 80 \times 10$ grid points, with the first cell centre a distance of $y^{+} \approx 1$ from the wall.

\subsection{Original Formulation with Fixed Relaxation Time-Scales $\&$ Manual Zone Selection}

To test the basic implementation, automatic relaxation time-scales and zone selection is disabled while consistency is enforced using the drift terms originally proposed by Xiao and Jenny [4]. Equations (14) and (16) are used to enforce consistency of the LES solution. The RANS momentum equation includes the drift term of Equation (19) and the Launder-Sharma $k-\varepsilon$ model is modified to include the drift terms of Equations (20) and (21). The time-scales are given the same fixed values as used by Xiao and Jenny: $\Upsilon=11 \delta / U_{b}, \gamma_{l 1}=\gamma_{r 1}=\gamma_{r 2}=1.3 \delta / U_{b}$ and $\gamma_{l 2}=0.67 \delta / U_{b}$. It is assumed that the LES is well-resolved in all cells with $y \geq 0.2 \delta$.

Results for the predicted friction Reynolds number are given in Table 1. The hybrid solver provides predictions that are in better agreement with the DNS data than both the pure LES and pure RANS solutions from the same respective grids; the pure LES prediction is particularly poor due to the deliberate under-resolution of its grid nearwalls.

\begin{tabular}{|c|c|}
\hline Solution & $\mathbf{R e}_{\tau}$ \\
\hline Nominal & 395.0 \\
\hline DNS & 395.8 \\
\hline Pure RANS & 384.5 \\
\hline Pure LES & 352.6 \\
\hline Hybrid LES/RANS & 387.9 \\
\hline
\end{tabular}

Table 1: Comparison of $R e_{\tau}$ predictions from the hybrid solver using the original Xiao and Jenny's [4] formulation. DNS data from [20, 21].

Mean streamwise velocity and turbulent kinetic energy profiles are shown in Figures 1 and 2. Profiles are shown for solutions extracted from the hybrid solver's RANS grid (denoted Hybrid RANS) and a pure RANS simulation on the same grid (Pure RANS), as well as that from the hybrid solver's LES grid (Hybrid LES) and a pure LES simulation on the same grid (Pure LES). The dual-mesh hybrid framework is designed to ensure that the hybrid LES and hybrid RANS solutions are consistent with each other. However inspecting velocity profiles in Figure 1(b) reveals that there are inconsistencies between the Hybrid LES and hybrid RANS solutions in the buffer layer; 


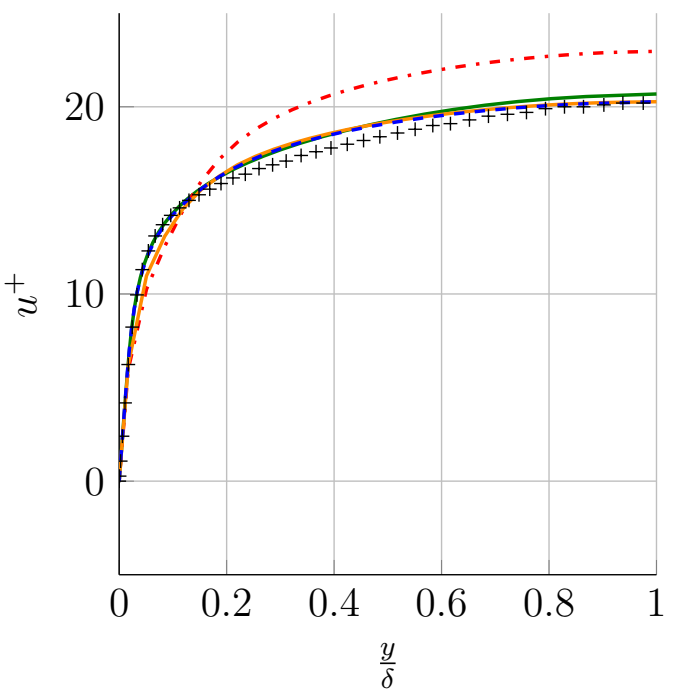

(a) Linear Scale

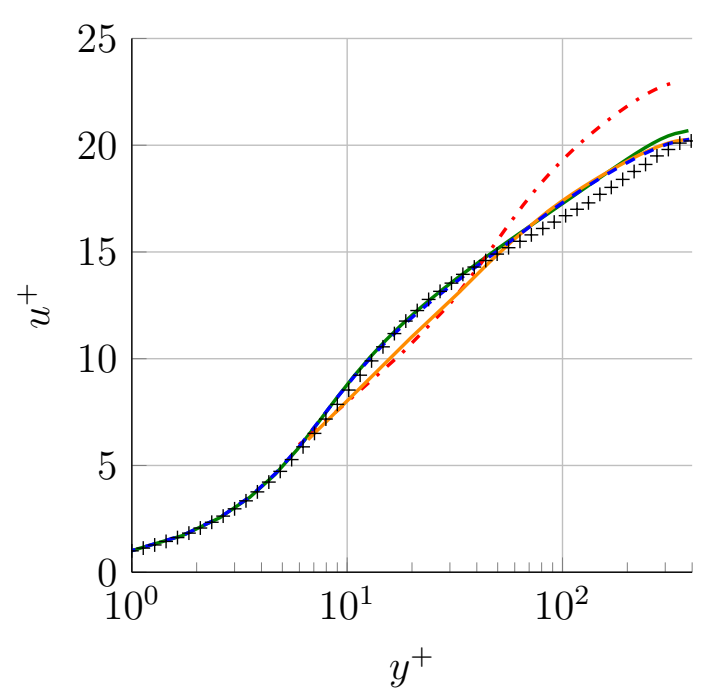

(b) Semi-log Scale

Figure 1: Mean velocity profiles in wall units for a $R e_{\tau}=395$ channel flow: + DNS [20, 21], — Pure RANS, . - - Pure LES, - - Hybrid RANS grid, — Hybrid LES grid.

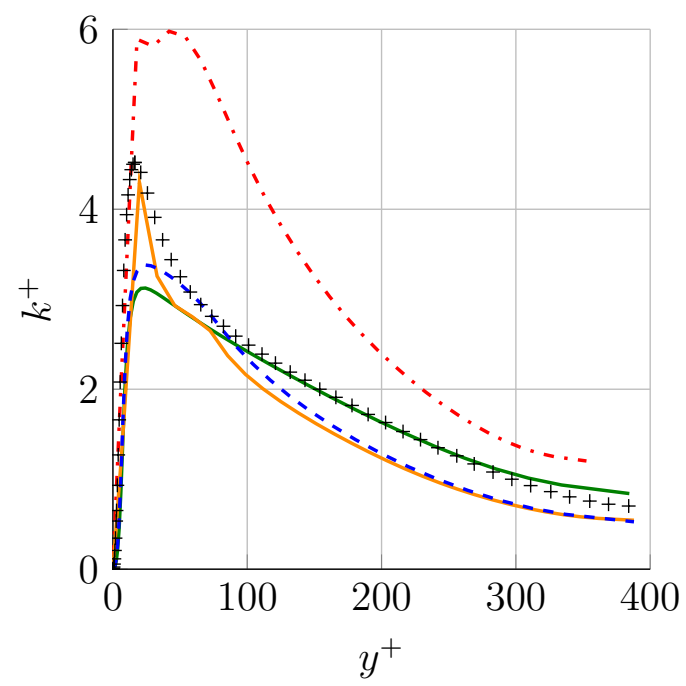

Figure 2: Mean turbulent kinetic energy profiles in wall units for a $R e_{\tau}=395$ channel flow: + DNS [20, 21], — Pure RANS, - - - Pure LES, - - Hybrid RANS grid, — Hybrid LES grid.

the hybrid LES grid does not predict the correct buffer layer behaviour while the RANS grid does.

In Figure 2, there is a large inconsistency between hybrid solutions from the two meshes for the peak in the turbulent kinetic energy at $y^{+} \approx 16$. A known defect of the Launder-Sharma $k-\varepsilon$ model is its inability to predict correctly this peak in $k$. In the near-wall region the LES solution is under-resolved and so it should be relaxed towards 
the locally incorrect RANS $k$ field; one would expect the result from the hybrid RANS \& LES grids to be consistent with each other but discrepant to the DNS data. The fact that here hybrid results from the LES grid are in better agreement with the DNS data than those from the RANS grid suggests that the framework is not effective at enforcing the consistency requirements throughout the entire domain.

\subsection{Revised Formulation with Automatic Relaxation Time-Scales 8 Zone Selection}

The previous results demonstrate that the original dual mesh hybrid LES/RANS framework is able to provide predictions that are in better agreement with DNS data than under-resolved LES on the same grid, though it has some shortcomings. There are a large number of case-specific free parameters which need to be specified, and the framework could be more effective at enforcing both the velocity and turbulent kinetic energy consistency requirements throughout the entire domain.

In the revised approach, LES $k$ consistency is enforced by adjusting both the resolved and modelled contributions to the EWA turbulent kinetic energy, using Equations (17) and (18). The RANS dissipation rate drift term is removed and consistency of RANS $k$ is enforced using the modified production term of Equation (24). Furthermore, the blending function in Equation (27) is used to activate the drift terms, while the relaxation time-scales are computed automatically using Equation (28).

Figure 3 demonstrates the action of the blending function which exhibits a smoother, delayed switchover compared to the step function approach of Xiao and Jenny. The manual zone selection method does not result in a perfect switchover at the specified wall distance of $y / \delta=0.2$ due to cell centres not being precisely colocated here. In some areas of the domain, $\sigma^{L}$ no longer takes an integer value of 0 or 1 . Here the drift terms are active in both meshes, and the solutions are simultaneously relaxed towards each other. The blending function can be thought of as adjusting the relaxation timescales and thus the rate at which one solution is relaxed towards the other. Recall that $\sigma^{R}=1-\sigma^{L}$ and so if for example $\sigma^{L}<0.5$ the LES solution will be relaxed towards the RANS solution faster than the RANS solution is relaxed towards the LES.

The mean relaxation time-scales are shown in Figure 4(a) and vary significantly throughout the domain. The RMS values of the relaxation time-scales are shown in Figure 4(b) and demonstrate how the time-scales vary in time to reflect the unsteadiness of the large scale turbulent structures. This is particularly apparent near the core of the channel, around $y / \delta \approx 0.85$, as a result of large scale turbulent structures moving between the upper and lower halves of the domain.

Figure 5 shows the mean velocity and turbulent kinetic energy profiles obtained using the new formulation. These results demonstrate that as well as providing a higher degree of automation, the modifications to the original dual-mesh hybrid LES/RANS framework improve the consistency between solutions from the two grids. In comparison to the results using the original formulation (Figure 1), the consistency of velocity predictions from the hybrid solver's RANS and LES grids in the buffer layer is significantly improved in Figure 5(a). Predictions from both hybrid grids are now correct in the buffer layer, whereas previously the LES grid predicted a premature log-layer. 


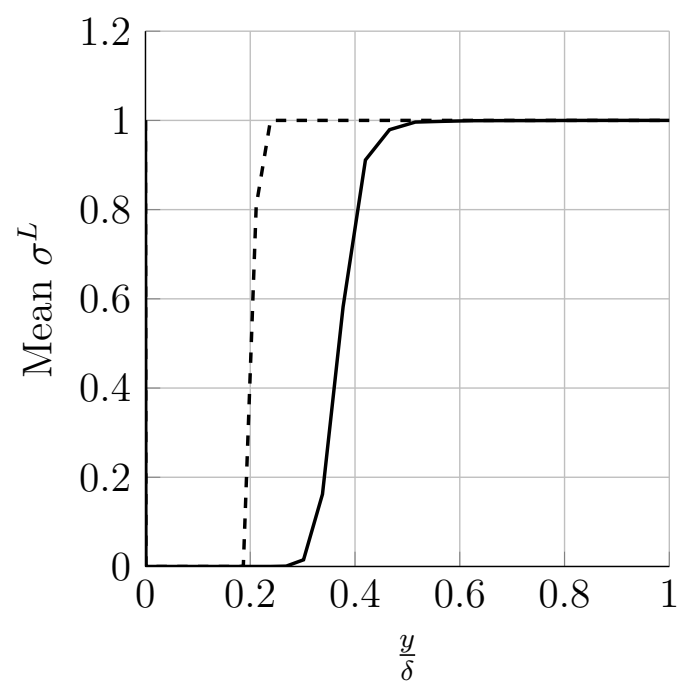

Figure 3: Mean of $\sigma^{L}$ from the step function proposed by Xiao and Jenny (- - - ) and from the blending function developed in the present work (-). A value of $\sigma^{L}=0$ corresponds to the LES drift terms being fully on while the RANS drift terms are fully off.

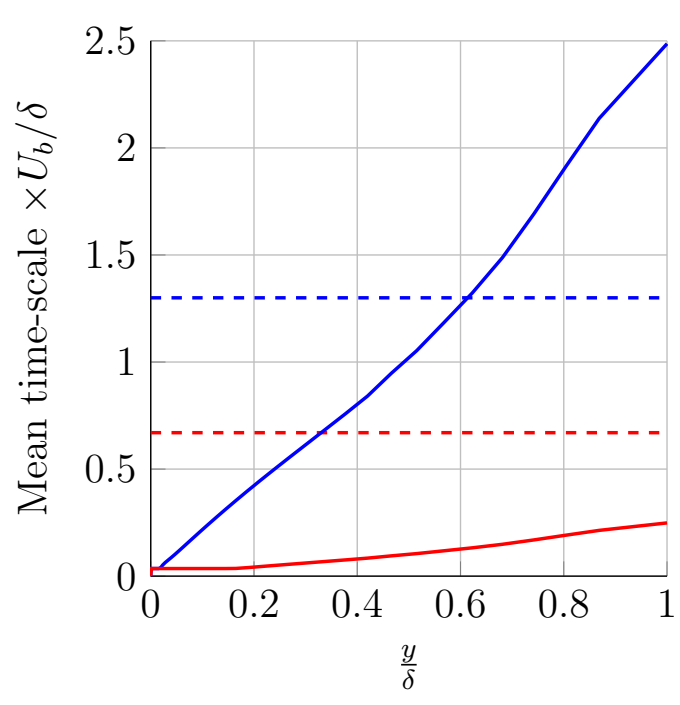

(a) Mean relaxation time-scales

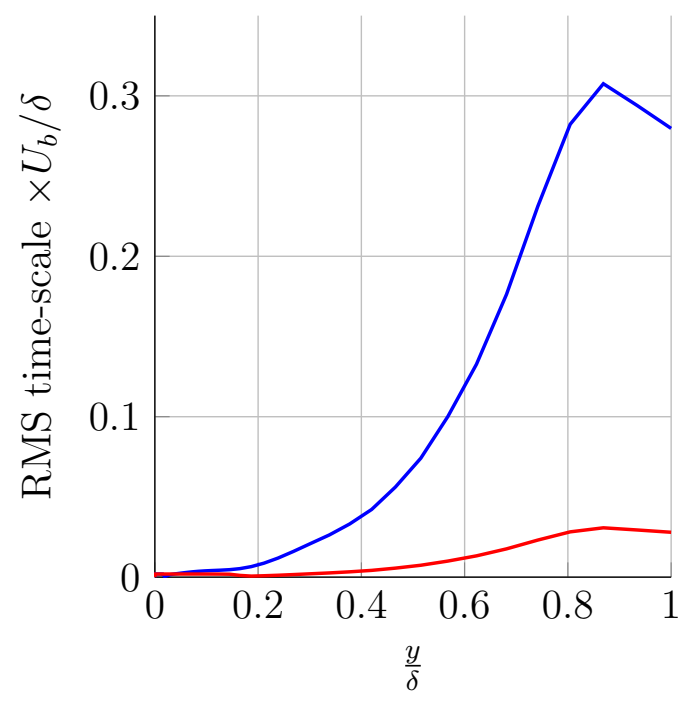

(b) RMS relaxation time-scales

Figure 4: Mean and RMS relaxation time-scales for a $R e_{\tau}=395$ channel flow: - - - fixed $\gamma_{l 1}=1.3 \delta / U_{b}$, - - - fixed $\gamma_{l 2}=0.67 \delta / U_{b}$, — variable $\gamma_{l 1}$ computed using Equation (28a) and — variable $\gamma_{l 2}$ computed using Equation (28b).

There is also improved internal consistency of the turbulent kinetic energy fields. Results in Figure 5(b) show very good levels of internal consistency throughout, whereas results from the original formulation (Figure 2) show a large inconsistency in the peak value of $k$ from the hybrid solver's RANS and LES grids. 


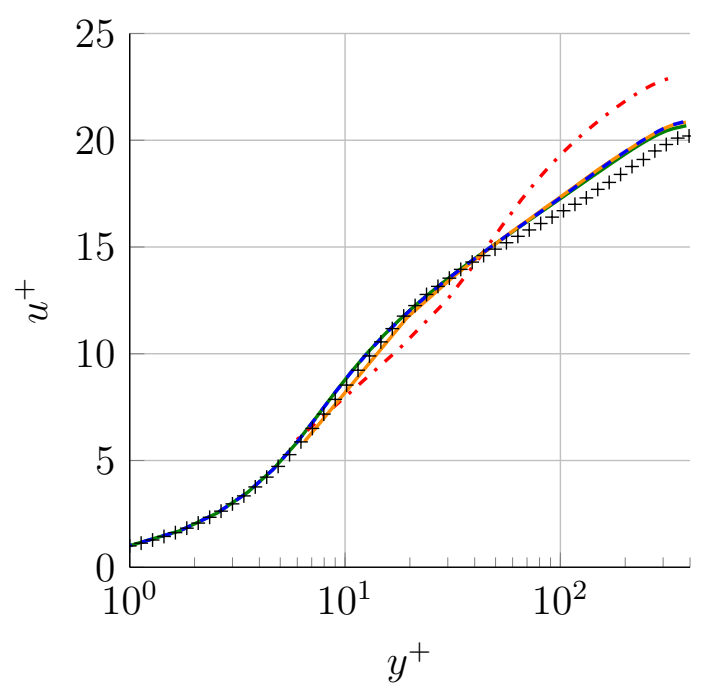

(a) Mean Velocity

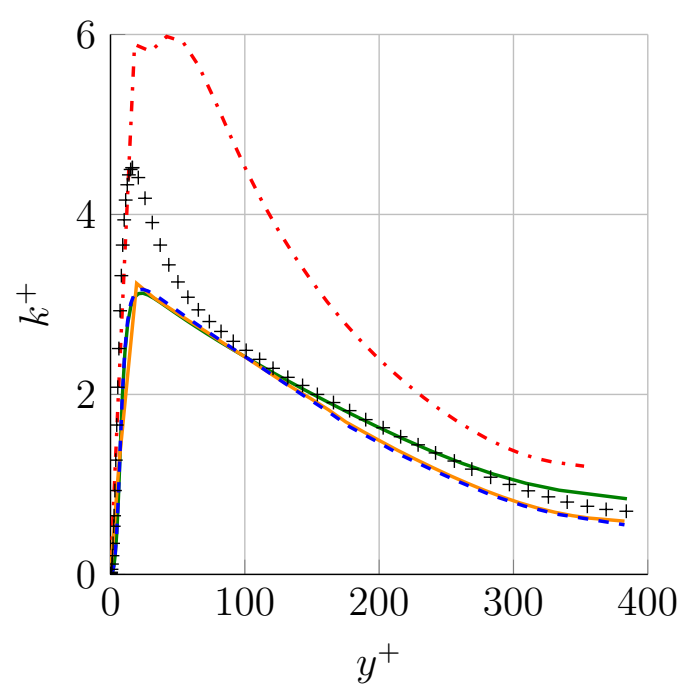

(b) Mean Turbulent Kinetic Energy

Figure 5: Mean velocity profiles in wall units for a $R e_{\tau}=395$ channel flow: + DNS [20, 21], — Pure RANS, - - - Pure LES, - - Hybrid RANS grid, — Hybrid LES grid. Here the hybrid solver uses automatic zone selection and variable relaxation time-scales.

\subsection{Use of an Improved RANS Model}

The LES is considered under-resolved in near-wall regions, and so the EWA of its solution is relaxed towards RANS predictions. However, a known defect of the LaunderSharma $k-\varepsilon$ model is its inability to predict the near-wall peak in $k$. We now rerun the previous simulation using the elliptic blending $k-\varepsilon-v^{2} / k$ RANS model, which is designed to provide improved near-wall predictions by accounting for wall-normal stress anisotropy. The only change to the model is the production term modification described in Equation (24), to enforce consistency of the turbulent kinetic energy field; the method is equally applicable to other eddy viscosity models which solve a transport equation for $k$.

\begin{tabular}{|c|c|}
\hline Solution & $\mathbf{R e}_{\tau}$ \\
\hline Nominal & 395.0 \\
\hline DNS & 395.8 \\
\hline Launder-Sharma Pure RANS & 384.5 \\
\hline$k-\varepsilon-v^{2} / k$ Pure RANS & 387.3 \\
\hline Pure LES & 352.6 \\
\hline Launder-Sharma Hybrid LES/RANS, $\Upsilon=11 \delta / U_{b}$ & 382.7 \\
\hline$k-\varepsilon-v^{2} / k$ Hybrid LES/RANS, $\Upsilon=11 \delta / U_{b}$ & 388.8 \\
\hline$k-\varepsilon-v^{2} / k$ Hybrid LES/RANS, $\Upsilon=44 \delta / U_{b}$ & 389.9 \\
\hline
\end{tabular}

Table 2: Comparison of $R e_{\tau}$ predictions from the revised hybrid solver using automatic zone selection and variable relaxation time-scales. DNS data from $[20,21]$. 


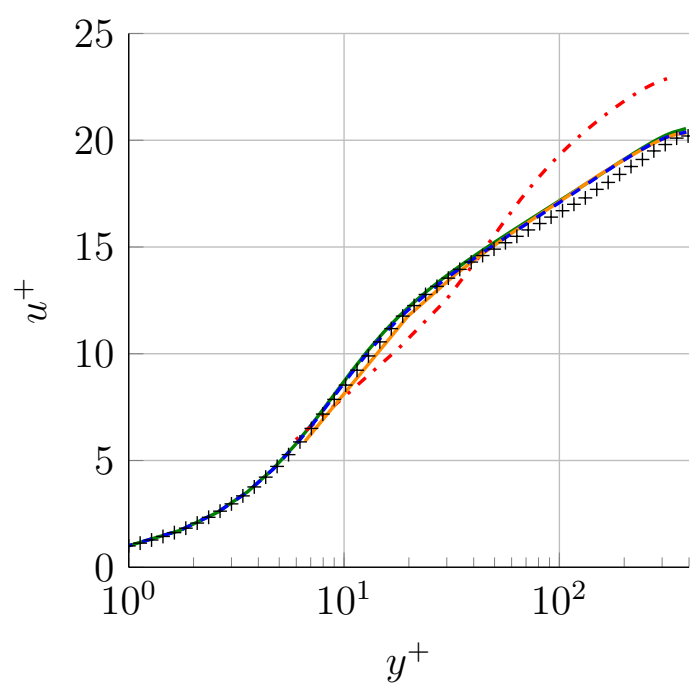

(a) Mean Velocity

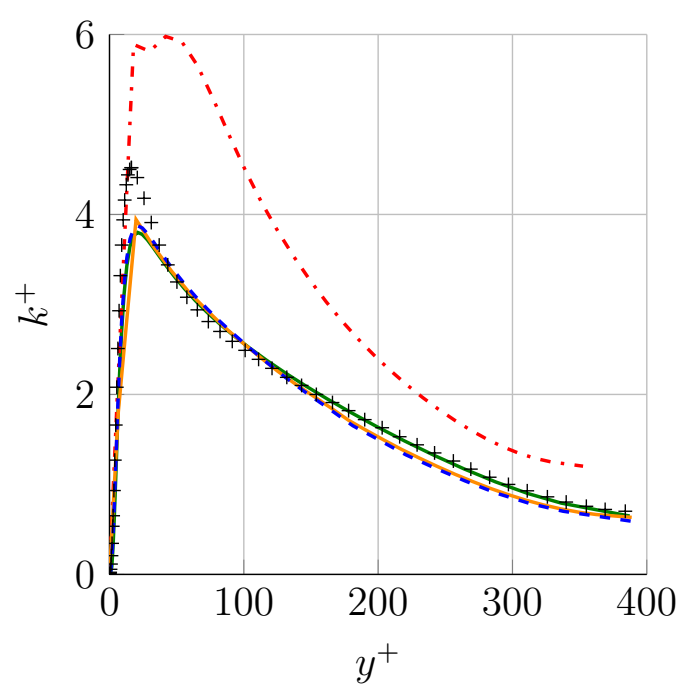

(b) Turbulent Kinetic Energy

Figure 6: Mean velocity and T.K.E profiles in wall units for a $R e_{\tau}=395$ channel flow using the elliptic blending $k-\varepsilon-v^{2} / k$ RANS model: + DNS [20, 21], — Pure RANS, - - - Pure LES, - - - Hybrid RANS grid, — Hybrid LES grid.

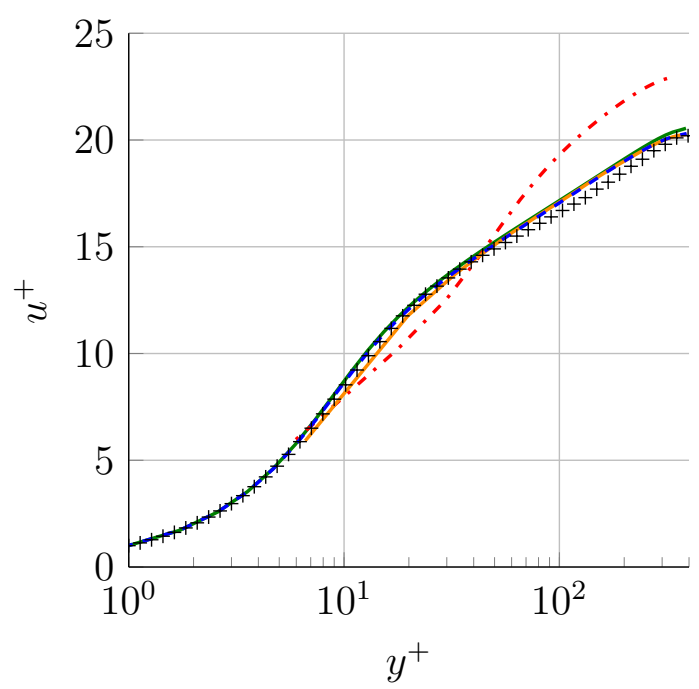

(a) Mean Velocity

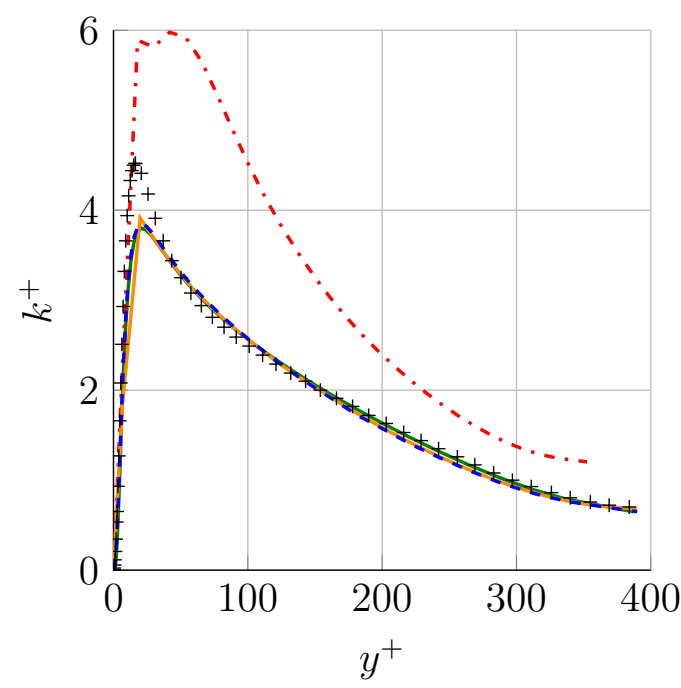

(b) Turbulent Kinetic Energy

Figure 7: Mean velocity and T.K.E profiles in wall units for a $R e_{\tau}=395$ channel flow using the elliptic blending $k-\varepsilon-v^{2} / k$ RANS model with an increased averaging time-scale $\Upsilon=44 \delta / U_{b}$ : + DNS [20, 21], — Pure RANS, - - - Pure LES, - - Hybrid RANS grid, — Hybrid LES grid.

Mean velocity and the turbulent kinetic energy predictions are shown in Figure 6. The improved near-wall modelling of the elliptic blending $k-\varepsilon-v^{2} / k$ model results in significantly better predictions for the near-wall peak in $k$, Figure $6(\mathrm{~b})$, for both pure 
RANS and dual-mesh hybrid LES/RANS simulations, compared to results using the Launder-Sharma model in Figure 5(b). Consistency between hybrid solutions from the two grids remains excellent throughout. Table 2 illustrates that changing the underlying RANS model has also improved the friction Reynolds number prediction by $1.6 \%$.

Only minor sensitivity to the averaging time-scale is observed. Increasing $\Upsilon$ by a factor of 4 results in a slightly more satisfactory $k$ profile in the log-layer, Figure $7(\mathrm{~b})$, and a small improvement in the $R e_{\tau}$ prediction, Table 2.

\section{Results for a $R e_{\tau}=1000$ Channel Flow}

To demonstrate that the blending function and automatic relaxation time-scales are effective at higher Reynolds numbers, we now consider a $R e_{\tau}=1000$ channel flow. Again the EWA time-scale is set to $\Upsilon=44 \delta / U_{b}$. The LES grid has $80 \times 60 \times 80$ grid points with constant spacing in the streamwise, wall-normal and spanwise directions, respectively. The first cell centre is a distance of $y^{+} \approx 6$ from the wall with $\Delta x^{+} \approx 80$ and $\Delta z^{+} \approx 40$; the LES mesh is again deliberately designed to be under-resolved in near-wall regions. The low-Reynolds number RANS grid has $25 \times 100 \times 25$ grid points, with a first cell wall distance of $y^{+} \leq 1$.

Like in the lower Reynolds number case, the revised dual-mesh hybrid LES/RANS prediction for the friction Reynolds number, Table 3, is in better agreement with DNS data than that from under-resolved LES on the same grid.

\begin{tabular}{|c|c|}
\hline Solution & $\mathbf{R e}_{\tau}$ \\
\hline Nominal & 1000.0 \\
\hline DNS & 999.4 \\
\hline Pure RANS & 991.6 \\
\hline Pure LES & 954.2 \\
\hline Hybrid LES/RANS & 1006.8 \\
\hline
\end{tabular}

Table 3: Comparison of $R e_{\tau}$ predictions using the improved dual-mesh hybrid LES/RANS framework. DNS data from $[22,23,24]$.

As Reynolds number increases the relative thickness of the boundary layer decreases. This causes the blending function, Figure 8(a), to place the switchover between forcing of the LES and RANS solutions closer to the wall when compared to the $R e_{\tau}=395$ case. Figure 8(b) demonstrates that the relative magnitude of the non-dimensionalised relaxation time-scales is also reduced as a consequence of the increased Reynolds number. These results demonstrate how the blending function and automatic relaxation time-scales allow the dual-mesh hybrid LES/RANS framework to adapt itself to the prevailing flow.

The consistency of mean velocity and turbulent kinetic energy profiles from the two hybrid grids is excellent throughout, as shown in Figure 9. The hybrid LES/RANS mean velocity profile is in very good agreement with DNS data; unlike in the pure LES solution, correct behaviour is predicted in the buffer layer. Hybrid LES/RANS predictions for the turbulent kinetic energy are also in far better agreement with DNS 


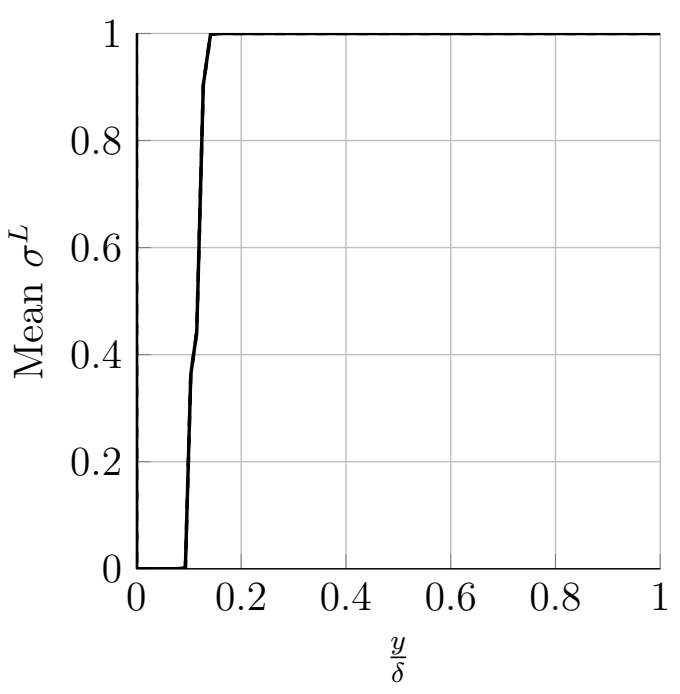

(a) Mean $\sigma^{L}$

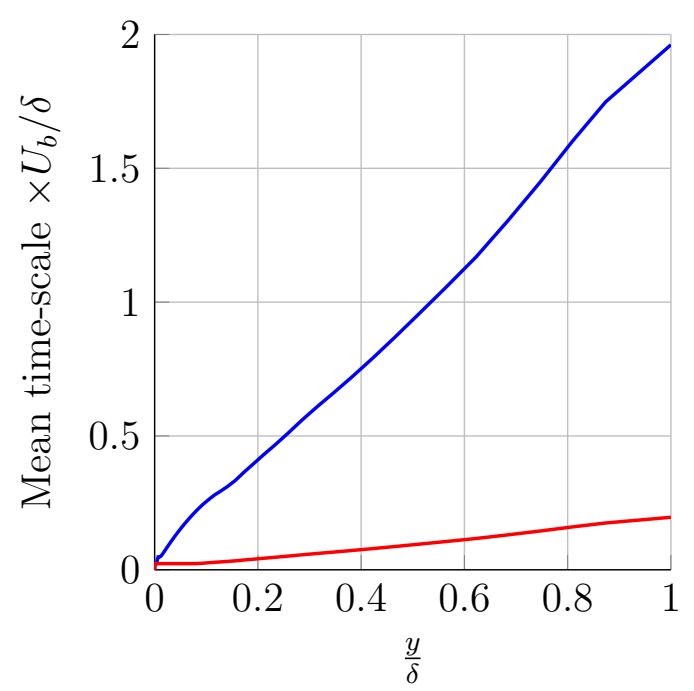

(b) Mean relaxation time-scales

Figure 8: Mean $\sigma^{L}$ and relaxation time-scales for a $R e_{\tau}=1000$ channel flow: $-\gamma_{l 1}$ computed using Equation (28a) and $-\gamma_{l 2}$ computed using Equation (28b).

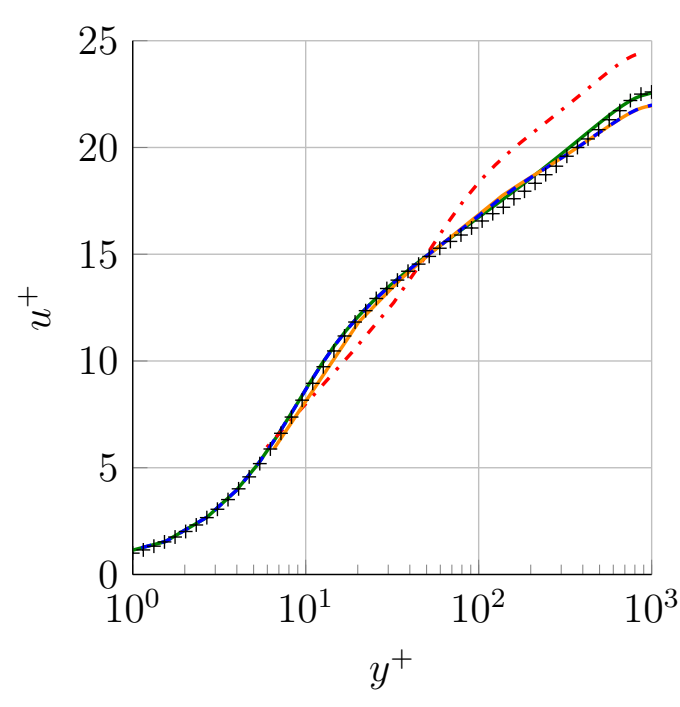

(a) Mean Velocity

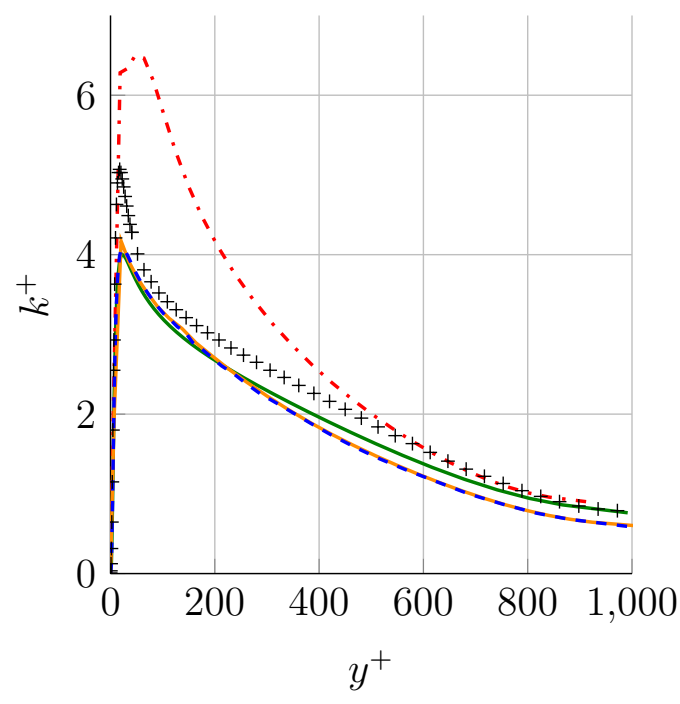

(b) Turbulent Kinetic Energy

Figure 9: Mean velocity and T.K.E profiles in wall units for a $R e_{\tau}=1000$ channel flow using the elliptic blending $k-\varepsilon-v^{2} / k$ RANS model : + DNS [22, 23, 24], — Pure RANS, - - - Pure LES, - - Hybrid RANS grid, — Hybrid LES grid.

data than pure LES. Agreement of $k$ with DNS data is perhaps a little worse than in the $R e_{\tau}=395$ case, which is a consequence of the RANS model under-predicting $k$ in the early stages of the log-layer. 


\section{Results for the Flow Through Periodic Hills}

We now use the improved hybrid LES/RANS framework developed above to study a $R e=H U_{\text {in }} / \nu=10,595$ flow through the periodic hills illustrated in Figure 10. The hills are of height $H$ and the bulk velocity at the hill crest is denoted by $U_{\text {in }}$. The computational domain has dimensions $9 H \times 3.036 H \times 4.5 H$. Periodic boundary conditions are used in the spanwise and streamwise directions, while a constant mass flow rate is imposed by a pressure gradient. Results are compared to predictions from a wall-resolved LES performed by Breuer et al. [25]. The case tests the ability of the method to predict separation on a curved surface and natural reattachment further downstream; the prediction of such phenomenon can be challenging for both RANS and wall-modelled LES approaches.

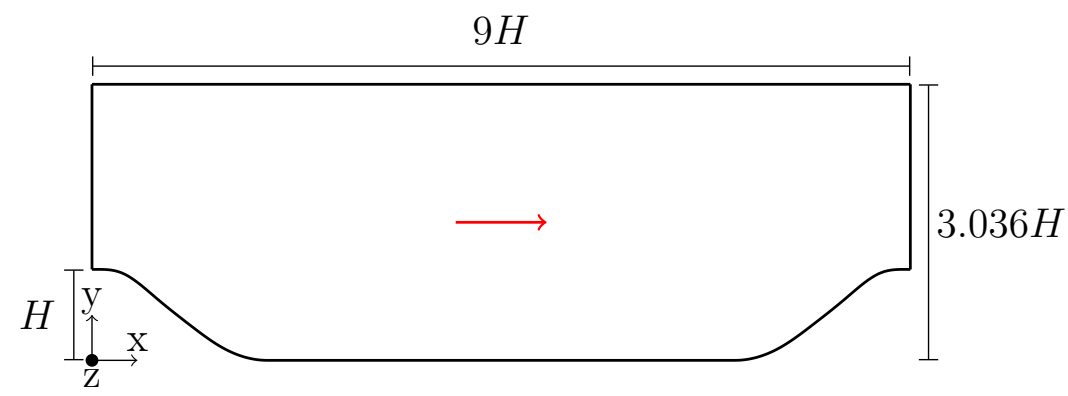

Figure 10: Geometry of the periodic hills. The depth of the domain in the spanwise direction is $4.5 H$ and the red arrow indicates the flow direction.

The computational grids are similar to those employed in [4]. The LES mesh has $74 \times 37 \times 36$ grid points while the RANS mesh contains $74 \times 37 \times 18$ grid points. The RANS mesh is designed to have a first cell wall spacing of $y^{+} \leq 1$ throughout, while in the LES mesh $y^{+} \approx 30$. Both of the meshes have uniform spacings in the spanwise and streamwise directions. The RANS and LES grids have growth ratios in the wall-normal direction of 1.2 and 1.0 , respectively.

Results are presented for the original dual-mesh hybrid LES/RANS framework proposed by Xiao and Jenny and from the version developed herein. When using the original coupling, the relaxation and averaging time-scales take the same values as in [4] $\left(\gamma_{l 1}=\gamma_{r 1}=\gamma_{r 2}=0.26 \mathrm{H} / U_{\text {in }}\right.$ and $\gamma_{l 2}=0.07 \mathrm{H} / U_{\text {in }}$ with $\left.\Upsilon=2.2 \mathrm{H} / U_{\text {in }}\right)$ and the LES is assumed to be well-resolved in all cells that are a distance more than $0.2 H$ from the nearest wall. The only parameter which needs to be specified in the improved dualmesh hybrid LES/RANS solver is the time-scale of the exponentially weighted average. $\Upsilon=18 H / U_{\text {in }}$ is found to be sufficient to ensure that the LES grid EWA fields do not contain features that are too fine to be accurately interpolated onto the RANS grid and results display little sensitivity to this parameter being increased further. The improved coupling computes the relaxation time-scales automatically and the drift terms are activated using the blending function in Equation (27). In both cases the elliptic blending $k-\varepsilon-v^{2} / k$ RANS model is employed. 
Mean $\sigma_{L}$ fields, which indicate where the LES drift terms are active, for the original and improved dual-mesh hybrid LES/RANS approaches are shown in Figure 11. Using the original coupling the LES drift terms are fully deactivated $\left(\sigma_{L}=1\right)$ beyond a wall-distance of $y / H=0.2$ throughout the entire domain. The blending function in Equation (27) is able to account for the local variation in the boundary layer thickness and as a result the wall distance at which the LES drift terms become fully deactivated varies when using the improved coupling. In much of the domain the LES drift terms are deactivated nearer to the wall when using the improved coupling, indicating that the RANS solution's influence is limited to a thinner layer.
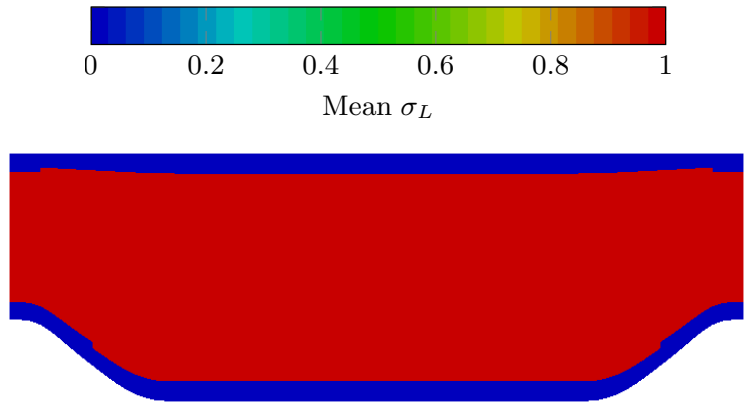

(a) Original Coupling

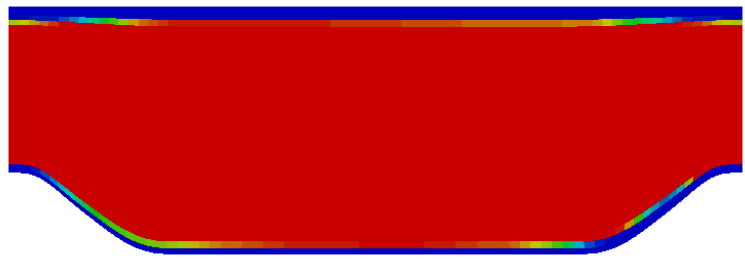

(b) Improved Coupling

Figure 11: Mean $\sigma_{L}$ for a $R e=10,595$ flow through periodic hills.

Mean streamwise velocity predictions using the original and revised coupling are shown in Figure 12. In both cases hybrid LES/RANS predictions are in better agreement with the benchmark data than pure LES on the same grid. Pure LES provides poor predictions in the separated region and in the upper wall boundary layer. This is a consequence of the grid being too coarse near-walls. The dual-mesh hybrid LES/RANS approach remedies this issue by using RANS to support the LES solution in these critical areas. Along the lower wall in the vicinity of the reattachment point, predictions from the revised framework, Figure 12(b), are in significantly better agreement with reference data than those from the original Xiao and Jenny coupling, Figure 12(a).

Turbulent kinetic energy profiles obtained using the original coupling are shown in Figure 13(a). Significant discrepancies between the hybrid RANS and LES grid $k$ predictions are visible near the upper wall. Here, forcing of the LES EWA velocity towards its RANS counterpart increases the strain-rate. The LES subgrid $k$ increases in response, such that its contribution to the EWA turbulent kinetic energy field dominates 


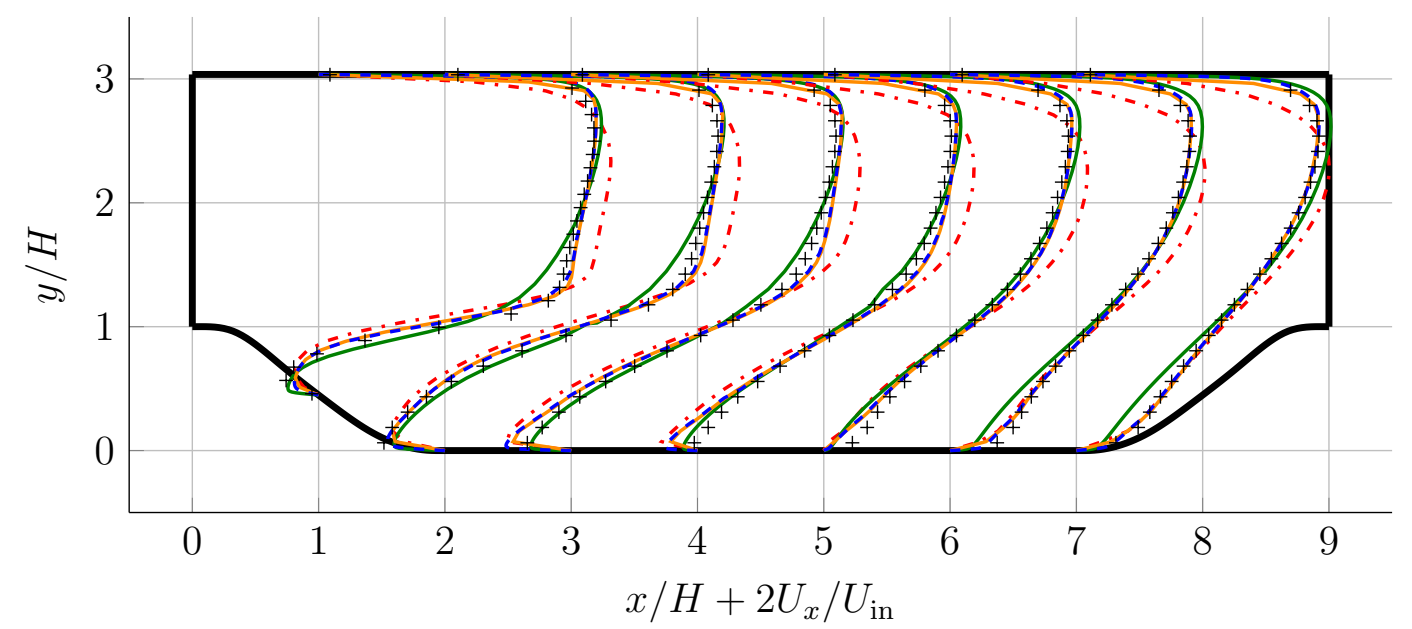

(a) Original Coupling

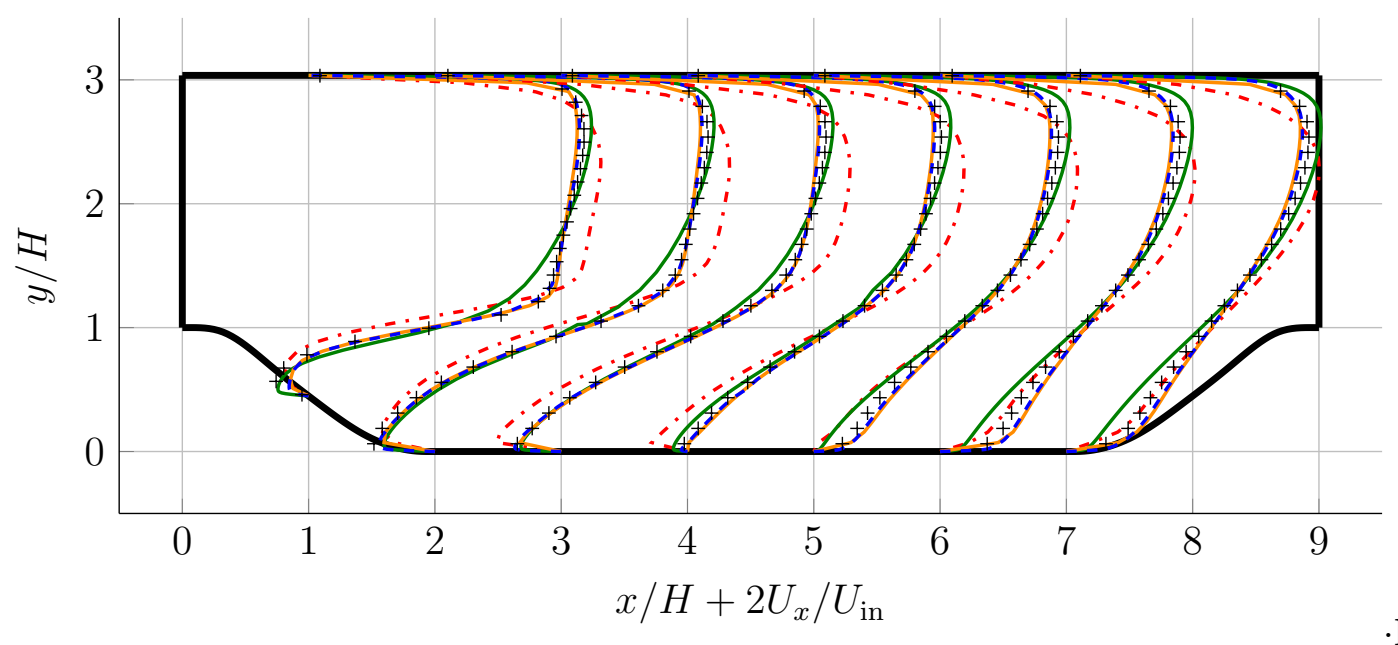

(b) Improved Coupling

Figure 12: Mean streamwise velocity profiles for a $R e=10,595$ flow through periodic hills: + ref. LES [25], — Pure RANS, - - - Pure LES, - - - Hybrid RANS grid, — Hybrid LES grid.

that of the resolved fluctuations. However, the original dual-mesh hybrid LES/RANS framework is only able to adjust the resolved fluctuations and so a consistent coupling between the turbulent kinetic energy fields cannot be established. This results in degraded near-wall $k$ predictions. Since the inconsistency also affects the unforced LES predictions further from the wall, which the RANS solution (and ultimately the nearwall LES solution) is reliant upon, the overall hybrid LES/RANS solution is degraded.

The consistency between hybrid LES and hybrid RANS grid predictions for $k$ using the revised framework is excellent throughout Figure 13(b), and the predictions are also in better agreement with the reference data than those using the original coupling. Enforcing $k$ consistency by adjusting both the resolved fluctuations and subgrid $k$ where the LES is under-resolved avoids the issue experienced by the original coupling along 


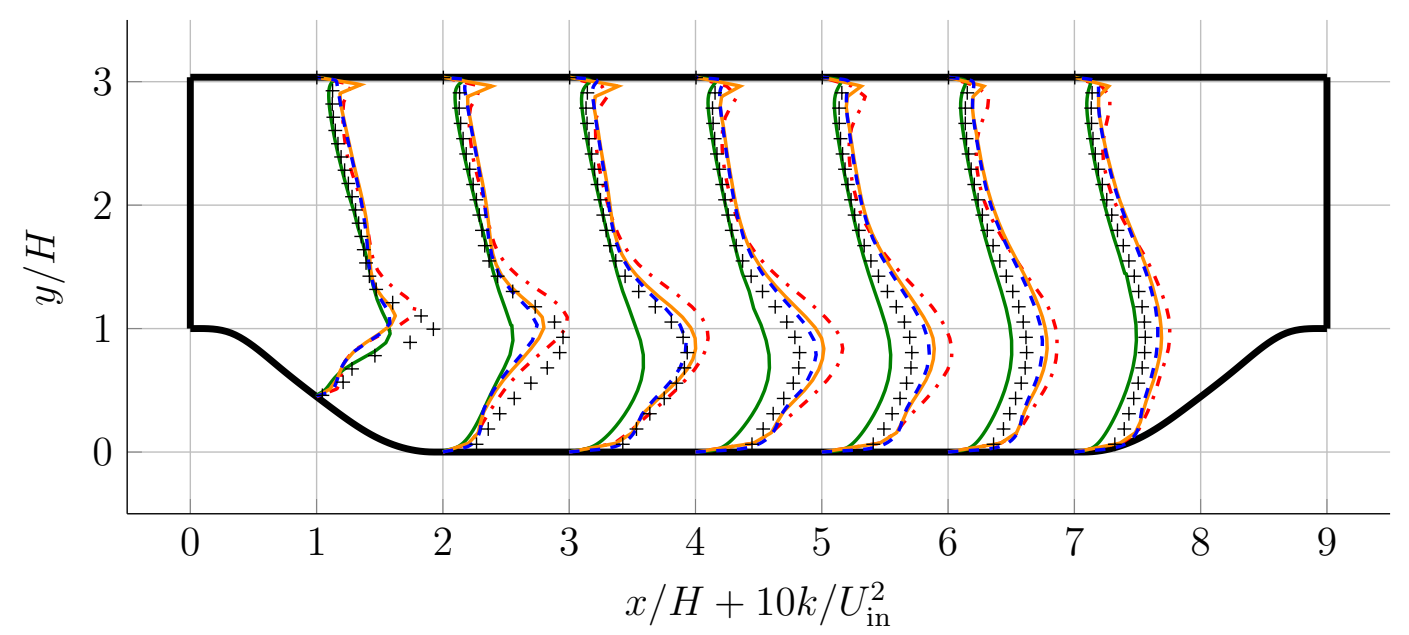

(a) Original Coupling

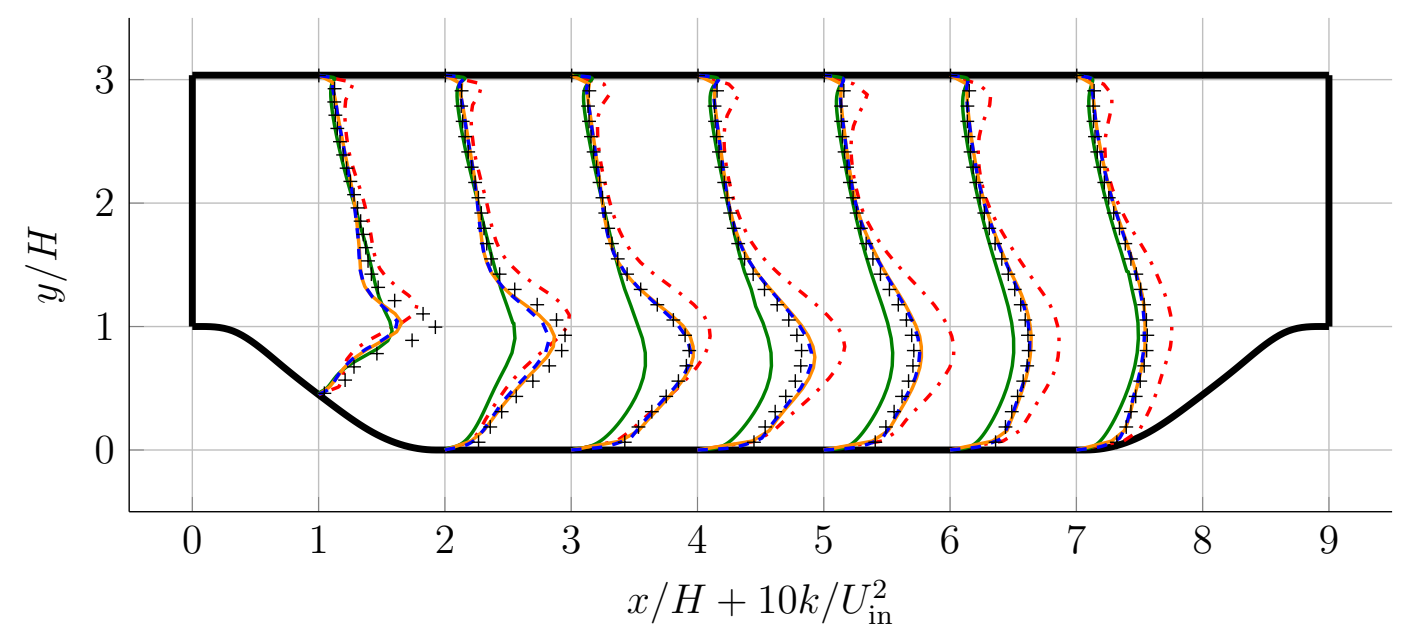

(b) Improved Coupling

Figure 13: Mean turbulent kinetic energy profiles for a $R e=10,595$ flow through periodic hills: + ref. LES [25], — Pure RANS, - - - Pure LES, - - Hybrid RANS grid, — Hybrid LES grid.

the upper wall. The only significant discrepancy between hybrid predictions and the reference data occurs at $X / H=1$. This is expected to be a consequence of the underlying RANS eddy viscosity model being unable to account for stress-strain misalignment associated with the separation point. 


\section{Conclusions}

A dual-mesh hybrid LES/RANS solver has been implemented in OpenFOAM 2.3.x. RANS and LES equations are solved on separate grids with drift terms to enforce consistency between the RANS and LES solutions. To avoid the fundamental inconsistencies associated with many unified hybrid LES/RANS approaches, where the LES is classified as well-resolved the RANS solution is relaxed towards an exponentially weighted average of the LES solution and where the LES is classified as under-resolved its EWA is relaxed towards the RANS solution.

Numerous improvements to the original proposal of Xiao and Jenny [4] have been developed, which aim to increase automation and improve the consistency between solutions from the two meshes:

- Where the LES is classified as under-resolved, consistency of the EWA turbulent kinetic energy is enforced by adjusting both its resolved and modelled contributions. In the original coupling only the resolved fluctuations are adjusted.

- Drift terms in the RANS turbulent kinetic energy and dissipation rate equations have been replaced by a modified production term.

- The drift terms are activated automatically using a blending function, which assumes that the LES solution should be supported by RANS in viscous affected regions. This is designed to avoid the near-wall grid refinement required by wallresolved LES.

- The relaxation time-scales are now formulated in terms of turbulent time-scales, they vary both spatially \& temporally to reflect the prevailing flow physics. Previously these case dependent parameters were user-specified constants.

Results for a $R e_{\tau}=395$ channel flow show that the hybrid framework provides predictions that are in better agreement with DNS data than pure LES and pure RANS on the same grids. The improvements to the framework developed in the present work result in excellent consistency between mean velocity and turbulent kinetic energy predictions from the hybrid solver's two grids, whereas considerable inconsistencies were apparent in near-wall regions when using the formulation of Xiao and Jenny. Agreement of hybrid predictions with DNS data is further improved by replacing the LaunderSharma $k-\varepsilon$ RANS model with the elliptic blending $k-\varepsilon-v^{2} / k$. The importance of choosing a sufficiently long averaging time-scale is also demonstrated. The improved coupling is also found to yield decent predictions for a $R e_{\tau}=1000$ channel flow.

Dual-mesh hybrid LES/RANS predictions for a $R e=10,595$ flow through periodic hills are also in better agreement with reference data than both pure RANS and pure LES on the same grids. Predictions using the revised coupling are in better agreement with reference data and the internal consistency of solutions from the two grids is also improved, when compared to the original method proposed by Xiao and Jenny. 
The present work has focussed on developing an automatic coupling between RANS and LES grids, such that RANS is used to support the LES solution in viscous affected near-wall regions. Predictions from this dual-mesh hybrid LES/RANS approach are in good agreement with reference data when using meshes that are far too coarse for wallresolved LES. In the cases reported, the CPU time required for the dual-mesh hybrid LES/RANS simulation is typically $\sim 30-50 \%$ greater than the pure LES simulation on the same grid. In order to maximise the computational cost savings that can be realised, further work should also focus on developing a dual-mesh decomposition technique that balances the MPI overhead associated with solving equations on each of the meshes in parallel with that of the mesh-to-mesh interpolation. A subsequent manuscript will present extensions of the approach for heat transfer problems.

\section{Acknowledgements}

The authors would like to thank Rolls-Royce for funding this work and acknowledge use of the Computational Shared Facility at the University of Manchester. This work also made use of the facilities at the N8 HPC Centre of Excellence, provided and funded by the N8 consortium and EPSRC (Grant No.EP/K000225/1). The Centre is

co-ordinated by the Universities of Leeds and Manchester. The authors also thank the reviewers for their helpful suggestions.

\section{References}

[1] S Chapuliot, C Gourdin, T Payen, J P Magnaud, and A Monavon. Hydro-thermalmechanical analysis of thermal fatigue in a mixing tee. Nuclear Engineering and Design, 235(5):575-596, 2005.

[2] R Tunstall, D Laurence, R Prosser, and A Skillen. Benchmarking LES with wallfunctions and RANS for fatigue problems in thermal-hydraulics systems. Nuclear Engineering and Design, 308:170-181, 2016.

[3] J Fröhlich and D von Terzi. Hybrid LES/RANS methods for the simulation of turbulent flows. Progress in Aerospace Sciences, 44(5):349-377, 2008.

[4] H Xiao and P Jenny. A consistent dual-mesh framework for hybrid LES/RANS modelling. Journal of Computational Physics, 231(4):1848-1865, 2012.

[5] H Xiao, Y Sakai, R Henniger, M Wild, and P Jenny. Coupling of solvers with nonconforming computational domains in a dual-mesh hybrid LES/RANS framework. Computers \&f Fluids, 88:653-662, 2013.

[6] H Xiao, J Wang, and P Jenny. Dynamic evaluation of mesh resolution and its application in hybrid LES/RANS methods. Flow, Turbulence and Combustion, 93(1):141-170, 2014. 
[7] P R Spalart, W H Jou, M Strelets, and S R Allmaras. Comments on the feasibility of LES for wings, and on a hybrid RANS/LES approach. Advances in DNS/LES, 1:4-8, 1997.

[8] L Davidson and S H Peng. Hybrid les-rans modelling: a one-equation sgs model combined with a $k-\omega$ model for predicting recirculating flows. International Journal for Numerical Methods in Fluids, 43(9):1003-1018, 2003.

[9] E Labourasse and P Sagaut. Reconstruction of turbulent fluctuations using a hybrid RANS/LES approach. Journal of Computational Physics, 182(1):301-336, 2002.

[10] C Meneveau, T S Lund, and W H Cabot. A Lagrangian dynamic subgrid-scale model of turbulence. Journal of Fluid Mechanics, 319:353-385, 1996.

[11] M Germano. Turbulence: the filtering approach. Journal of Fluid Mechanics, 238:325-336, 1992.

[12] A Yoshizawa and K Horiuti. A statistically-derived subgrid-scale kinetic energy model for the large-eddy simulation of turbulent flows. Journal of the Physical Society of Japan, 54(8):2834-2839, 1985.

[13] C Fureby, G Tabor, HG Weller, and AD Gosman. A comparative study of subgrid scale models in homogeneous isotropic turbulence. Physics of Fluids (1994present), 9(5):1416-1429, 1997.

[14] B Andersson, R Andersson, L Håkansson, M Mortensen, R Sudiyo, and B Van Wachem. Computational fluid dynamics for engineers. Cambridge University Press, 2011.

[15] B de Laage de Meux, B Audebert, R Manceau, and R Perrin. Anisotropic linear forcing for synthetic turbulence generation in large eddy simulation and hybrid RANS/LES modelling. Physics of Fluids (1994-present), 27(3):035115, 2015.

[16] OpenFoam. http://www.openfoam.org, accessed: 01/02/2016.

[17] R I Issa. Solution of the implicitly discretised fluid flow equations by operatorsplitting. Journal of Computational Physics, 62(1):40-65, 1986.

[18] B E Launder and B I Sharma. Application of the energy-dissipation model of turbulence to the calculation of flow near a spinning disc. Letters in Heat and Mass transfer, 1(2):131-137, 1974.

[19] F Billard and D Laurence. A robust $k-\varepsilon-\overline{v^{2}} / k$ elliptic blending turbulence model applied to near-wall, separated and buoyant flows. International Journal of Heat and Fluid Flow, 33(1):45-58, 2012. 
[20] K Iwamoto, Y Suzuki, and N Kasagi. Database of fully developed channel flow. THTLAB Internal Reports, Dept of Mech Eng, The Univ of Japan, 2002.

[21] K Iwamoto, Y Suzuki, and N Kasagi. Reynolds number effect on wall turbulence: toward effective feedback control. International Journal of Heat and Fluid Flow, 23(5):678-689, 2002.

[22] J Graham, K Kanov, X Yang, M Lee, N Malaya, C Lalescu, R Burns, G Eyink, A Szalay, R Moser, and C Meneveau. A Web services accessible database of turbulent channel flow and its use for testing a new integral wall model for LES. Journal of Turbulence, 17(2):181-215, 2016.

[23] Y Li, E Perlman, M Wan, Y Yang, C Meneveau, R Burns, S Chen, A Szalay, and $\mathrm{G}$ Eyink. A public turbulence database cluster and applications to study lagrangian evolution of velocity increments in turbulence. Journal of Turbulence, (9).

[24] E Perlman, R Burns, Y Li, and C Meneveau. Data exploration of turbulence simulations using a database cluster. In Proceedings of the 2007 ACM/IEEE conference on Supercomputing.

[25] M Breuer, N Peller, Ch Rapp, and M Manhart. Flow over periodic hills-numerical and experimental study in a wide range of Reynolds numbers. Computers $\&$ Fluids, 38(2):433-457, 2009. 